\title{
Sensing Hazards with Operational Unmanned Technology
}

Adapted from "NOAA's Sensing Hazards with Operational Unmanned Technology (SHOUT) Experiment Observations, and Forecast Impacts" by Gary A. Wick (NOAA/Earth System Research Laboratory), Jason P. Dunion, Peter G. Black, John R. Walker, Ryan D. Torn, Andrew C. Kren, Altug Aksoy, Hui Christophersen, Lidia Cucurull, Brittany Dahl, Jason M. English, Kate Friedman, Tanya R. Peevey, Kathryn Sellwood, Jason A. Sippel, Vijay Tallapragada, James Taylor, Hongli Wang, Robbie E. Hood, and Philip Hall. Published online in $B A M S$, July 2020. For the full, citable article, see https://doi.org/10.1175 /BAMS-D-18-0257.1.

\footnotetext{
NASA's Global Hawk instrumented * for the SHOUT campaigns. Key payloads include the High-Altitude Monolithic Microwave Integrated Circuit (MMIC) Sounding Radiometer (HAMSR), High-Altitude Imaging Wind and Rain Airborne Profiler (HIWRAP),

Airborne Vertical Atmospheric Profiling System (AVAPS) dropsonde launcher, and Lightning Instrument Package (LIP) sensors. (Photo courtesy NASA).
}

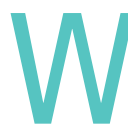
hile significant progress has been made in forecasting highimpact weather like tropical cyclones, skill remains limited. High-altitude, long-endurance (HALE) unmanned aircraft systems (UAS) like NASA's Global Hawk may complement and enhance the observations that could improve these forecasts. While the capabilities of the Global Hawk have been successfully demonstrated for research, the potential impacts of its observations on operational weather forecasting need to be established.

HALE UAS could, for example, temporarily supplement a satellite observation gap, such as was feared might occur as the Suomi National Polar-Orbiting Partnership (Suomi NPP) awaited the deployment of its successor, Joint Polar Satellite System-1 (JPSS-1). To mitigate such risks, NOAA initiated the Sensing Hazards with Operational Unmanned Technology (SHOUT) project to demonstrate and test a prototype UAS in 2015-16. With no satellite data gap occurring, the project ultimately did

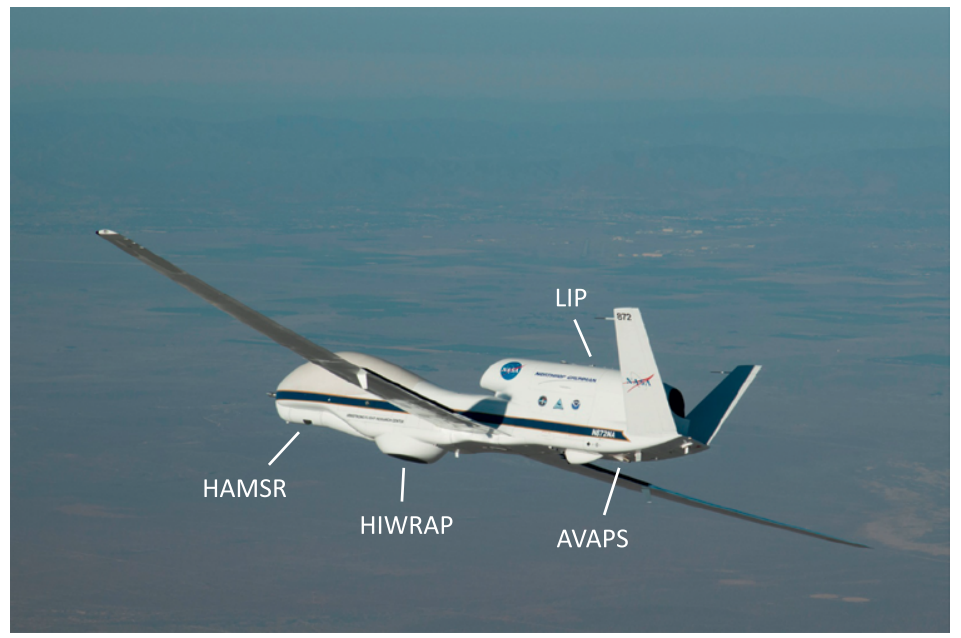



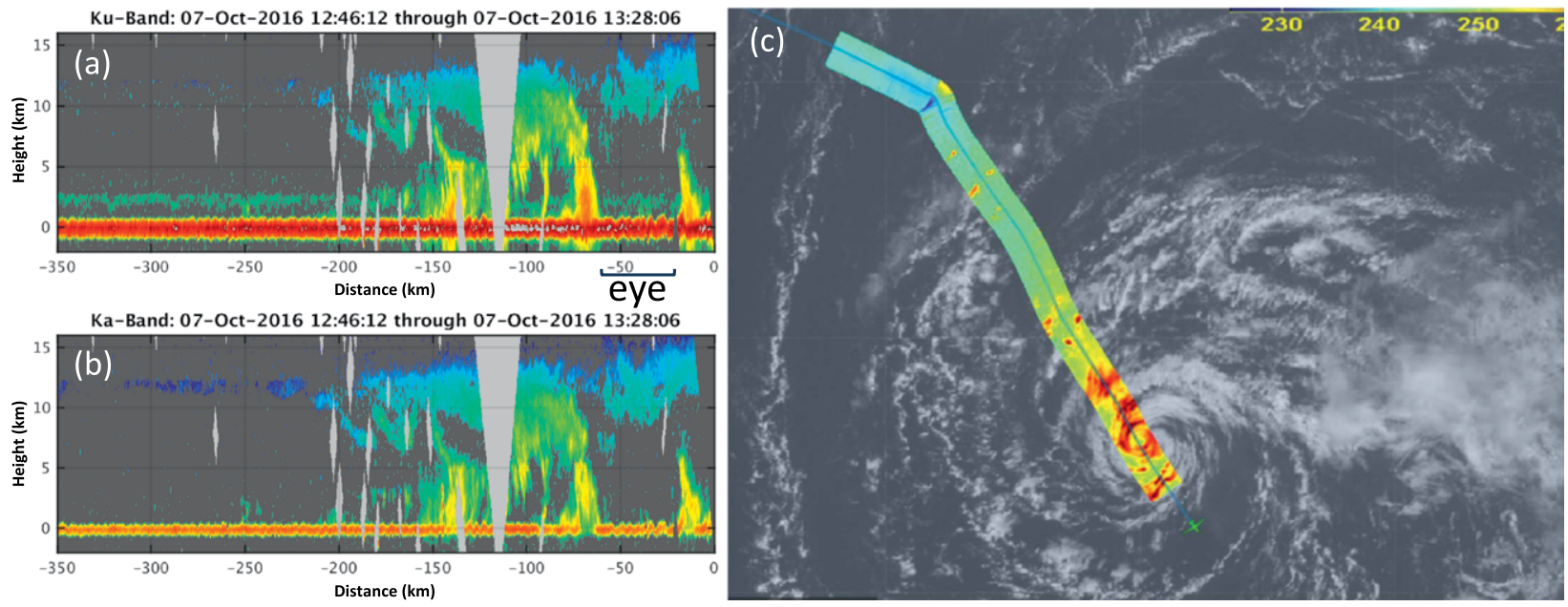

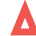

* Example of real-time remotely sensed imagery obtained from the NASA GH during SHOUT. (a),(b) Reflectivity cross sections from the HIWRAP during a center crossing over Hurricane Matthew. The position of the eye at the time of the overpass is noted on the images, and the eyewall reflectivity is clearly visible. The $x$ axis represents distance from the aircraft's location. (c) Real-time 50.3-GHz microwave brightness temperature from the HAMSR during an overpass of Tropical Depression Fred on 5 Sep 2015. The microwave signature is highly complementary to and correlated with the visual satellite reflectance from GOES-East displayed as the background. HIWRAP imagery courtesy G. Heymsfield at NASA Goddard; HAMSR image provided by S. Brown at NASA JPL.

not fully address the ability of the observations to mitigate a satellite gap, but instead focused on the broader benefit of UAS data to complement existing observing systems.

In SHOUT, NOAA partnered with NASA and focused on quantifying the impact of UAS observations on high-impact weather prediction through data impact studies using observing system experiments (OSEs), based on observations collected during prototype operational field missions, and observing system simulation experiments (OSSEs), based on simulated observing capabilities. The project also assessed the operational effectiveness of UAS with a cost-benefit analysis.

Such assessments require a large amount of representative observations to input into the forecast system. While OSSEs can test impacts from simulated observations, data denial studies with actual observations remain an important component of complete evaluations.

NASA's Global Hawk was utilized in all SHOUT field campaigns for multiple reasons. Key aircraft capabilities are its flight duration of roughly $24 \mathrm{~h}$, operating altitudes of 16,765-19,810 m (55,000$65,000 \mathrm{ft})$, and payload capacity of $680 \mathrm{~kg}(1,500$ pounds). Its in situ and remote sensing instrumentation, including dropwindsondes, radar, and radiometer, measured pressure, temperature, moisture, precipitation, winds, and electric fields both within storms and in their surrounding environments. Instrument communications and data return were made available via both Iridium and Ku-band systems. High data rates provided through Ku-band satellite communications facilitate real-time data utilization, even for larger-volume data streams from remote sensors housed in the Global Hawk, which cruises at approximately $620 \mathrm{~km} \mathrm{~h}^{-1}$ (335 kt).

The three successful SHOUT Global Hawk field observing campaigns support ongoing data impact studies. The campaigns sampled tropical cyclones as well as landfalling Pacific winter storms with their strong wind- and extreme precipitation-inducing atmospheric rivers. Targeting strategies during each campaign blended objective and subjective guidance into each flight plan. The methods focused on identifying the regions of greatest forecast sensitivity-where the forecast outcome (typically position and intensity for tropical cyclones) is most sensitive to environmental conditions and thus where it might be advantageous to deploy additional observations.

During the 2015 Hurricanes, 2016 El Niño Rapid Response (ENRR), and 2016 Hurricane Rapid Response (HRR) campaigns, the Global Hawk proved to be an effective platform for addressing SHOUT scientific objectives. The instruments were generally reliable (except for some early issues with the dropsonde launcher), and the adaptive sampling techniques for targeting dropsonde observations proved effective. Data collected during SHOUT have been extensively used in impact studies for both tropical cyclones and winter storms. National Hurricane Center forecasters 

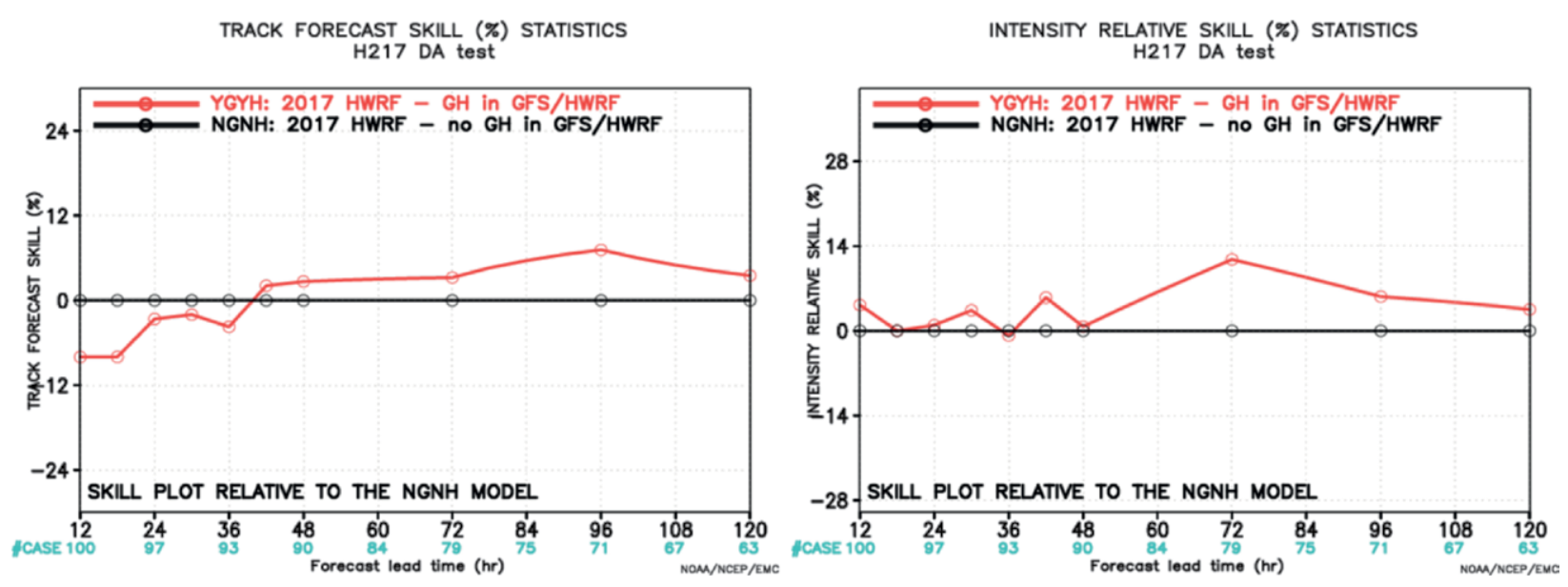

$\Delta$

* Average impact of GH dropsondes on operational HWRF forecasts of track and intensity for the $\mathbf{2 0 1 6}$ storms sampled by the GH (i.e., Gaston, Hermine, Karl, and Matthew). The results from adding GH dropsondes (red traces; coded YGYH) indicate improvements in the forecast skill for (left) track and (right) intensity relative to control runs performed assimilating all conventional observations including reconnaissance aircraft (black traces; coded NGNH).

used the data in real time, explicitly mentioning the Global Hawk HRR dropsonde data in 10 different discussions spanning the four tropical cyclones studied in 2016. These included a case where Gaston was upgraded to a hurricane based on Global Hawk data and other instances where the data provided key storm characteristics when other aircraft were unable to readily sample the storms.

While the limited number of storms analyzed makes it difficult to establish statistical significance, the studies consistently demonstrate the potential for forecast benefits from assimilating the Global Hawk's targeted observations during high-impact weather. The results obtained at NOAA's Environmental Modeling Center with different versions of

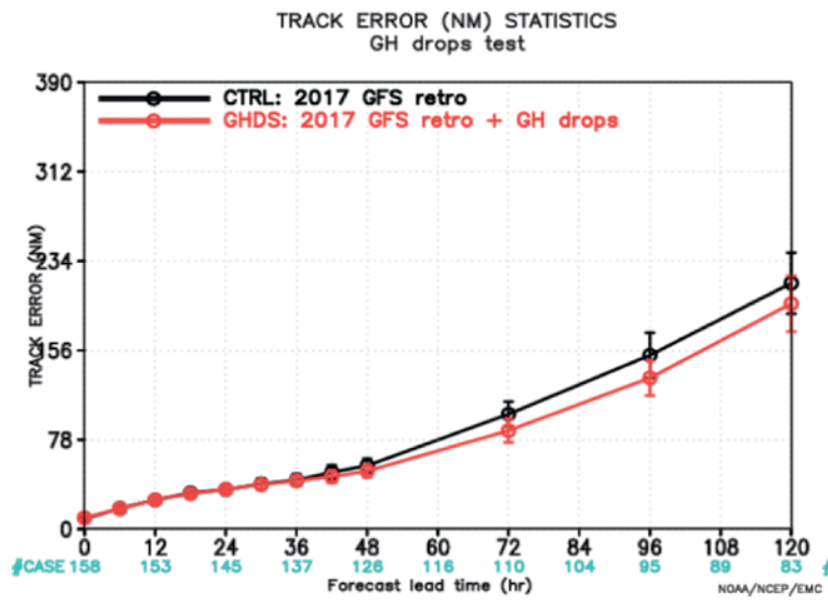

Impact of GH dropsonde measurements (GH drops) on the track error of North Atlantic storms within the 2017 operational GFS model. The results represent an average over the forecast cycles spanning from the first Gaston flight through the end of Gaston and the first Karl flight through the end of Matthew. All storms during these periods are incorporated, whether or not they were directly sampled by the GH. (left) Track error with (GHDS; red) and without (CTRL; black) the GH dropsondes. (right) Corresponding impact of the GH dropsondes on track forecast skill. $\frac{*}{\nabla}$ the operational modeling systems-both regional and global-are highly positive and argue for the potential merit of these unique observations.

Ongoing studies are continuing to expand the analyses to make the greatest possible use of the observations. These include increasing sample sizes by incorporating more observed storms, including from the collaborative Hurricane and Severe Storm Sentinel (HS3) and Eastern Pacific Origins and Characteristics of Hurricanes (EPOCH) campaigns. Studies are also using additional forecast models, and more fully evaluate the impact of the remotely sensed data. The

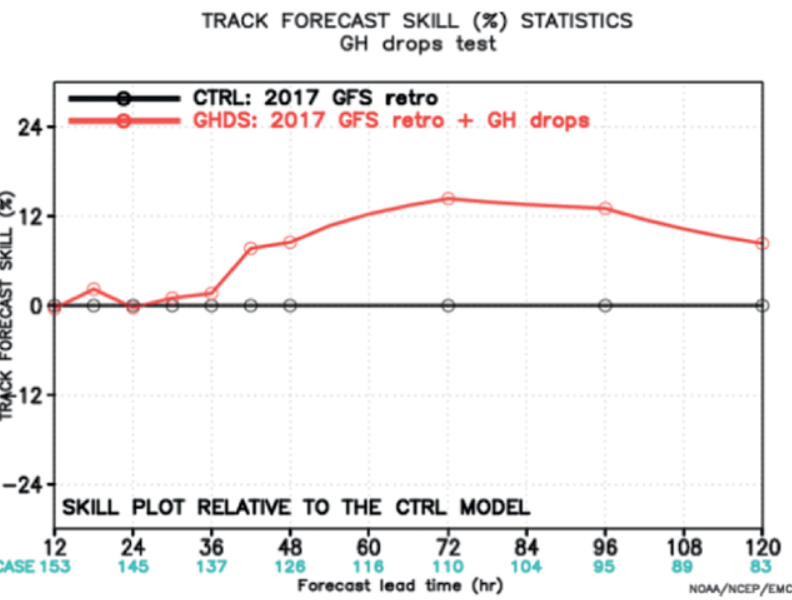


coverage of the observations offers potential that has yet to be fully exploited. One objective of these studies is to better understand which Global Hawk observations make the largest forecast impactsfor example, determining if observations from regions with increased forecast sensitivity indeed make a greater improvement in the forecasts.

Fully demonstrating forecast impacts ultimately requires observations of more storms over a longer period. Tests over fixed deployment periods are inevitably limiting, but are a necessary first step to justifying further investigation. Presently, there are no immediate plans for NOAA to employ a HALE UAS operationally, but the potential for scientific benefit is broadly supported by the success of SHOUT campaigns and the positive impacts of Global Hawk observations on numerical forecasts of high-impact weather.

In the near term, one of the greatest legacies of SHOUT is the application of new targeting approaches. The lessons learned from SHOUT are being applied to refined tasking of traditional aircraft in NOAA's operational tropical cyclone synoptic surveillance missions. The methods developed and tested during SHOUT have evolved into a NOAA Joint Hurricane Testbed project and were used semioperationally by the National Hurricane Center to develop high-altitude G-IV flight patterns.

\section{$\equiv$ METADATA}

BAMS: What would you like readers to learn from this article?

\section{Gary Wick (NOAA/Earth System Research Laboratory): Global Hawk} can provide highly useful observations of high-impact weather events that would be very difficult to obtain with any other existing aircraft or observing system.

BAMS: In this case, how is the usefulness determined?

GW: The utility of the observations is demonstrated by their potential for positive forecast impact. While it isn't possible to demonstrate statistically significant results from limited field campaigns such as SHOUT, the results were very encouraging in that it was possible to consistently see at least the potential for forecast benefit across a wide range of models when the data were added to our full existing observing system.

BAMS: How did you become interested in operational use of the Global Hawk?

GW: As someone involved in the NOAA Unmanned Aircraft Systems (UAS) Program for many years, I had long been curious about the potential for a platform such as the Global Hawk to provide novel data for many diverse aspects of weather and forecasting. I was fortunate to have participated in both the NASA-led Genesis and Rapid Intensification (GRIP) and Hurricane and Severe Storm Sentinel (HS3) campaigns through my involvement with the Global Hawk dropsonde system and was able to observe the potential application of the Global Hawk to tropical cyclone research. Those campaigns, however, didn't allow for a focus on operational hurricane forecasting. At the NOAA UAS Program we were extremely excited when support from the Disaster Relief Act of 2013 gave us the opportunity to conduct a dedicated campaign to examine the impact of observations from the Global Hawk on forecasts of high-impact weather events.

BAMS: What surprised you the most about your findings?

GW: As someone whose personal work hadn't centered around atmospheric models, assimilation, and weather forecasting, it surprised me early on how providing weather models with more high-quality, direct observations wouldn't necessarily improve the resulting forecasts. In some cases, it could actually degrade them. One might naively think that better data could only lead to a better final product. After gaining an appreciation of how challenging it is to achieve meaningful forecast improvements through addition of any data to our current observing and assimilation systems, I was very pleasantly surprised that the highly unique observations enabled by the Global Hawk still have the potential to help us improve our forecasts of high-impact weather events.

BAMS: What was the biggest challenge you encountered in this project?
GW: As with seemingly any field project, our biggest challenge was probably obtaining the weather events we were hoping to study during the necessarily limited duration of the campaign. Through the multiple years of the GRIP, HS3, and SHOUT campaigns, the Global Hawk developed almost a reputation as a "hurricane repellent" due to the limited number of storms. The state of EI Niño in the first year of SHOUT in 2015 also seemingly helped to reduce the number of candidate missions.

BAMS: But "repellent" finally wore off?

GW: Fortunately, we were able to extend the campaign. Perhaps the most interesting storm sampled during SHOUT, Hurricane Matthew in 2016, actually occurred after the scheduled end of the experiment. When conditions finally did present themselves, there was always also the challenge of having suitable local weather conditions to launch and recover the Global Hawk.

BAMS: How will you follow up?

GW: Unfortunately, we don't have the opportunity to fly additional Global Hawk missions in the immediate future, but we are still eager to more completely understand the impact of all the observations that have been collected. Several additional studies are underway to better evaluate the impact of all the different observations collected in different and the most recent models. 